\title{
CARDIOVASCULAR RISK IN TYPE 2 DIABETIC PATIENTS WITH ASYMPTOMATIC HYPERURICEMIA AND GOUT
}

\author{
R. Gancheva', A. Kundurdjiev², T. Kundurdjiev³ , Zl. Kolarov \\ ${ }^{1}$ Clinic of Rheumatology, University Hospital Sv. Iv. Rilski - Sofia, Bulgaria \\ ${ }^{2}$ Clinic of Nephrology, University Hospital Sv. Iv. Rilski - Sofia, Bulgaria \\ ${ }^{3}$ Faculty of Public Health, Medical University - Sofia, Bulgaria
}

\begin{abstract}
Aim: To study the differences in cardiovascular risk between type 2 diabetic and non-diabetic patients with asymptomatic hyperuricemia and gout using the Framingham Risk Score (FRS) and complex multimodal ultrasonography. Patients and methods: A total of 201 patients participated, divided into two groups: 1/ patients with asymptomatic hyperuricemia $(n=52)$, and 2/ patients with gout $(n=149)$. FRS was determined as well as ultrasound parameters, independent predictors of cardiovascular risk: left atrial size (LA), intima-media thickness (IMT) and common carotid artery resistive index (CCARI). Results: The patients in the two groups were age-matched and conventional cardiovascular risk factors were equally distributed. In the asymptomatic hyperuricemia group, 12 patients (23.1\%) had diabetes. In this group, there was no difference in FRS between diabetic and non-diabetic individuals. However, diabetic patients had larger LA, thicker intima-media and higher CCARI. In the gout group 18 subjects (12\%) had diabetes, but the FRS, LA, IMT and CCARI values were similar among diabetic and non-diabetic patients. Furthermore, when gout subjects were subdivided according to the presence of tophi, we found that the subgroup having gouty tophi and diabetes had larger LA ( $p=0.014)$ compared to those with gouty tophi without diabetes. Conclusion: In diabetic patients with asymptomatic hyperuricemia and gouty tophi, a more complex approach for estimation of cardiovascular risk is needed. Our work suggests that diabetes and tophi might potentiate their action on the cardiovascular system.
\end{abstract}

Key words: cardiovascular risk; type 2 diabetes; asymptomatic hyperuricemia; tophi

Corresponding author: R. Gancheva, MD, e-mail: rada_ga@mail.bg

\section{INTRODUCTION}

out is the most common cause of inflammatory arthritis in men [1]. Approximately $33 \%$ of gout patients have type 2 diabetes (T2D). Hyperuricemia and gout are closely related to the presence of metabolic syndrome. It is well documented that hyperuricemia precedes hyperin- sulinemia and it has been determined as a separate risk factor for the development of hyperinsulinemia and T2D [2]. There is evidence that gout and T2D share common risk alleles [3, 4]. In 1969 Boyle and McKiddie demonstrated for the first time that blood glucose and plasma insulin increased significantly in gout patients after glucose loading [5]. In 2006 the Finnish Diabetes Prevention Study reported that 
in middle-age high risk individuals, serum uric acid and its fluctuations are connected to the development of T2D [6]. In 2008 Choi analyzed the data of men with high cardiovascular risk in the Multiple Risk Factor Intervention Trial (MRFIT) and found that men with gout were at a higher risk of developing T2D. This relationship did not depend on the presence of known risk factors, but they should be treated aggressively for gout [7]. Later, Kim in 2015, and Rho in 2016 reaffirmed the high risk of developing T2D in subjects with gout $[8,9]$. The latest data in the field show that the presence of diabetes may prevent from development of gouty arthritis. Higher levels of glycated hemoglobin protect from symptoms of gout inflammation, which could be due to the uricosuric effect of glucose [10]. The risk factors for both diseases are similar and the pathogenic pathways are also shared [11]. There have been reports that urate-lowering therapy might reduce the risk for T2D [12].

Considering this well documented links between the phenotypes, we decided to look whether there might be a difference in the cardiovascular risk, calculated by the Framingham Risk Score (FRS) and additionally assessed by complex multimodal ultrasonography, in patients with asymptomatic hyperuricemia and gout with or without T2D. By ultrasound we measured the left atrial size (LA), intima-media thickness (IMT) and common carotid artery resistive index (CCARI) - parameters which are known as independent predictors of cardiovascular risk [13, 14]. In addition, we investigated changes in the end-diastolic volume index (EDVi), end-systolic volume index (ESVi), stroke volume index (SVi), ejection fraction $(E F)$, fractional shortening (FS) and left ventricular mass index (LVMi).

\section{METHODS}

This is a single center cross-sectional study, including 52 asymptomatic patients with hyperuricemia and 149 patients with gout (their clinical data are summarized in Table 1). Consecutive patients between June 2013 and May 2016 were included. Subjects with asymptomatic hyperuricemia were outpatients, while patients with gout were hospitalized at the University Rheumatology Clinic Sv. Ivan Rilski in Sofia. Indications for hospitalization were either gout attacks or assessment for further treatment. All the patients with gout fulfilled the 1977 ACR criteria [15]. The subcutaneous tophi on physical examination were typical
Gouty tophi. Subjects with asymptomatic hyperuricemia had serum uric acid above the normal range and no history of gout flare. The study protocol has been approved by the ethics committee of the institution. All participants gave informed consent in accordance with the Declaration of Helsinki.

The Framingham Risk Score (FRS) was calculated by an internet-based formula. It assessed the individual 10-year risk of developing cardiovascular disease incorporating 7 items such as sex, age, total cholesterol, high density lipoprotein cholesterol, smoking, the systolic blood pressure, and the presence of treated and untreated arterial hypertension. According to this scoring system, individuals with low 10 year-risk have $<10 \%$ coronary heart disease risk, those with intermediate risk $-10-20 \%$ and those with high risk $-\geq 20 \%[16,17]$. The clinical and laboratory assessments included current smoking status, presence of arterial hypertension defined as systolic blood pressure $\geq 140 \mathrm{mmHg}$, diastolic blood pressure $\geq 90$ $\mathrm{mmHg}$ or use of antihypertensive medication; diabetes mellitus, based on the current examination and/or documented use of insulin and/or oral hypoglycemic agents; dyslipidemia, based on elevated fasting lipid levels or low HDL and/or documented use of lipidlowering agents; reduced glomerular filtration rate defined as eGFR $<90 \mathrm{ml} / \mathrm{min}$ by the Cockroft-Gault formula, chronic kidney failure (CKF) as defined in the K/DOQI 2002 guidelines [18]. The additional laboratory parameters included in the analysis were: Creactive protein (CRP), serum creatinine, blood urea nitrogen (BUN), serum uric acid and serum albumin concentrations. Patients were defined as having sustained a cardiovascular event if they had proven coronary artery disease, cerebrovascular disease and/ or peripheral artery disease [19]. The exclusion criteria were: history of malignancy, exacerbated cardiac or renal failure, dilatative or hypertrophic cardiomyopathy, severe valvulopathy, atrial fibrillation, arterial blood pressure $>140 / 90 \mathrm{mmHg}$ and heart rate $<50$ $\mathrm{bpm}$ or $>90 \mathrm{bpm}$ during the ultrasonographic measurement.

Subjects underwent a multimodal ultrasound examination, performed by a single operator, who was unaware of the clinical data. From June 2013 till November 2015 the ultrasound examinations were carried out on an ALOKA-SSD-4000 device. Since November 2015 till May 2016 they were performed on a Philips HD 11 machine. The software of both devices had comparable characteristics. 
Echocardiographic examinations were conducted according to the recommendations of the American Society of Echocardiography with a $2.5 \mathrm{MHz}$ transducer phased array [20]. A minimum of three cardiac cycles were analyzed and averaged. From the parasternal long axis view, uniaxial anteriorposterior dimension of the left atrium (LA) was determined. End-diastolic volume index (EDVi), endsystolic volume index (ESVi), stroke volume index (SVi) and fractional shortening (FS) were recorded in 2-dimentionally guided M-mode. The Ejection fraction (EF) was calculated by the Teicholz method. The Left ventricular muscle mass was estimated by the Devereux formula. The carotid arteries were examined with a $10 \mathrm{MHz}$ linear transducer with $5 \mathrm{MHz}$ pulse Doppler frequency. The Intimamedia thickness (IMT) was bilaterally recorded in the far wall of the common carotid arteries $1-2 \mathrm{~cm}$ proximal to the bifurcation. The common carotid artery resistive index (CCARI) reflects the rigidity and distensibility of the common carotid arteries. It was determined with a pulse Doppler probe volume of $3 \mathrm{~mm}$ and an orientation angle between $30^{\circ}$ and $60^{\circ}$, and calculated as follows: [peak systolic velocity $(C \vee p)$ - end-diastolic velocity $(C V d) /$ peak systolic velocity (CVp)] [14]. The mean values of the clinical, laboratory and ultrasound parameters were analyzed.

\section{STATISTICS}

Statistical analyses were done by using SPSS version 13.0 (SPSS Inc., Chicago, IL, USA). The means with SDs and percentages were calculated to describe the clinical and ultrasound characteristics of the patients. Intergroup comparisons were performed by One-Sample Kolmogorov-Smirnov, Shapiro-Wilk and Paired-Samples T-tests. Proportional differences were tested using the chi-square and Fisher's exact test. All tests were two tailed, and $p$ values less than 0.05 were considered statistically significant.

\section{RESULTS}

The characteristics of our patients' cohort are summarized in Table 1. All examined patients in both subgroups were age-matched. There was a male preponderance in the gout group $(p<0.001)$. In the latter the level of hyperuricemia $(p=0.028)$, CRP $(p=0.022)$ and the number of smokers ( $p$ $=0.005)$ was higher. The remaining cardiovascular risk factors were equally distributed among the groups. According to the FRS, the cardiovascular risk was higher in subjects with gout than in asymptomatic hyperuricemia. Gout patients had an intermediate risk (mean \pm SD; $14.60 \pm 8.96 \%$ ) while those with asymptomatic hyperuricemia were scored as having a low risk (mean \pm SD; $5.69 \pm$ $6.03 \%$ ), (Table 1 ). The proportion of hypertensive patients and individuals who had suffered a cardiovascular event was larger in diabetic patients with asymptomatic hyperuricemia. Similarly, in the gout group the number of patients who had sustained a cardiovascular event was higher among individuals with T2D (Table 2).

In asymptomatic hyperuricemia the scores obtained by FRS were comparable between diabetic and nondiabetic patients $(p=0.580)$. However, subjects with T2D had larger LA (mean \pm SD; $41.08 \pm 4.64 \mathrm{~mm}$ vs $37.43 \pm 5.71 \mathrm{~mm}, p=0.023$ ), thicker intima-media (mean $\pm \mathrm{SD} ; 0.88 \pm 0.09 \mathrm{~mm}$ vs $0.77 \pm 0.18 \mathrm{~mm}, \mathrm{p}$ $=0.004)$ and higher CCARI (mean \pm SD; $0.71 \pm 0.03$ vs $0.68 \pm 0.06, p=0.036$ ), (Fig. $1 a, b, i, j$ ). No difference was registered in the mean values of EDVi, ESVi, SVi, EF, FS and LVMI (Fig. 1 c, d, e, f, g, h) between diabetic and non-diabetic individuals in this group. Likewise, among diabetic and non-diabetic individuals suffering from gout, there was no difference in the mean values of the FRS $(p=0.849)$, but EF of the heart was lower in those with diabetes $(p$ $=0.014$ ), (Fig. $2 \mathrm{a}, \mathrm{f}$ ). Among the diabetic and nondiabetic subjects with gout, the means of LA, EDVi, ESVi, SVi, EF, FS, LVMI, IMT and CCARI were simi$\operatorname{lar}$ (Fig. 2 b, c, d, e, g, h, i, j). When the gouty tophi group was examined separately the values of FRS were similar (Fig. 3 a). We observed that LA was larger in the cases with T2D (mean \pm SD; $45.41 \pm$ $5.62 \mathrm{~mm}$ vs $39.77 \pm 5.78 \mathrm{~mm}, \mathrm{p}=0.014$ ), (fig $3 \mathrm{~b}$ ). In this subgroup diabetics also had lower EDVi, ESVi, EF, FS and greater LVMI (Fig. $3 \mathrm{c}, \mathrm{d}, \mathrm{f}, \mathrm{g}, \mathrm{h}$ ). As to the parameters of the carotid arteries, there was no difference between diabetic and non-diabetic subjects with tophi, (Fig. $3 \mathrm{i}, \mathrm{j}$ ). 
Table 1. The baseline characteristics of the patients are shown

\begin{tabular}{|c|c|c|c|c|c|c|}
\hline \multirow{3}{*}{\begin{tabular}{|l} 
Index \\
Age (years) \\
\end{tabular}} & \multicolumn{2}{|c|}{ Asymptomatic hyperuricemia subgroup } & \multicolumn{2}{|c|}{ Gout subgroup } & \multirow{2}{*}{\multicolumn{2}{|c|}{$\mathrm{p}$-value }} \\
\hline & \multirow{2}{*}{$\begin{array}{c}\mathrm{N}(\%) \\
52\end{array}$} & \multirow{2}{*}{$\begin{array}{c}\text { Mean (SD) } \\
55.23(15.94)\end{array}$} & \multirow{2}{*}{$\begin{array}{c}\mathbf{N}(\%) \\
149 \\
\end{array}$} & \multirow{2}{*}{$\begin{array}{c}\text { Mean (SD) } \\
57.43(11.80)\end{array}$} & & \\
\hline & & & & & 0.365 & $\mathrm{t}$ \\
\hline Sex (males) & $29(55.8)$ & & $131(87.9)$ & & $<0.001$ & $x$ \\
\hline $\mathrm{BMI}\left(\mathrm{kg} / \mathrm{m}^{2}\right)$ & 52 & $31.10(6.55)$ & 149 & $30.02(5.12)$ & 0.227 & $\mathrm{t}$ \\
\hline $\mathrm{Hb}(\mathrm{g} / \mathrm{l})$ & 52 & $142.04(17.61)$ & 145 & $141.86(16.62)$ & 0.947 & $t$ \\
\hline$H t(g / l)$ & 50 & $0.42(0.05)$ & 143 & $0.42(0.06)$ & 0.428 & $U$ \\
\hline Serum level of uric acid $(\mu \mathrm{mol} / \mathrm{l})$ & 52 & $460.89(63.70)$ & 147 & $489.52(113.75)$ & 0.028 & $t$ \\
\hline BUN (mmol/l) & 36 & $5.99(2.38)$ & 98 & $6.99(3.89)$ & 0.301 & U \\
\hline eGFR (ml/min) & 52 & $111.03(51.46)$ & 149 & $106.43(49.50)$ & 0.568 & $\mathrm{t}$ \\
\hline $\mathrm{CRP}(\mathrm{mg} / \mathrm{l})$ & 34 & $6.53(7.44)$ & 131 & $17.22(27.20)$ & 0.022 & $U$ \\
\hline $\mathrm{HbA} 1 \mathrm{C} \%$ & 38 & $4.44(1.08)$ & 104 & $4.55(0.89)$ & 0.544 & $t$ \\
\hline $\mathrm{TC}(\mathrm{mmol} / \mathrm{l})$ & 46 & $5.63(1.21)$ & 131 & $5.41(1.20)$ & 0.287 & $t$ \\
\hline $\mathrm{HDL}(\mathrm{mmol} / \mathrm{l})$ & 44 & $1.22(0.29)$ & 113 & $1.14(0.37)$ & 0.017 & $U$ \\
\hline LDL (mmol/l) & 41 & $3.43(1.10)$ & 107 & $3.49(1.16)$ & 0.802 & $\mathrm{t}$ \\
\hline VLDL (mmol/l) & 38 & $0.75(0.31)$ & 102 & $0.93(0.51)$ & 0.134 & U \\
\hline TG (mmol/l) & 46 & $1.90(0.85)$ & 131 & $1.97(1.16)$ & 0.570 & U \\
\hline FRS (\%) & 52 & $5.69(6.03)$ & 149 & $14.60(8.96)$ & $<0.001$ & $U$ \\
\hline Dyslipidemia & $38(86.4)$ & & $11(94.9)$ & & 0.091 & $x$ \\
\hline $\mathrm{BMI}\left(>30 \mathrm{~kg} / \mathrm{m}^{2}\right)$ & $22(42.3)$ & & $67(45.0)$ & & 0.740 & $x$ \\
\hline Arterial hypertension & $35(67.3)$ & & $119(80.4)$ & & 0.054 & $x$ \\
\hline $\begin{array}{l}\text { Patients with a previous cardiovas- } \\
\text { cular event }\end{array}$ & $8(15.4)$ & & $23(15.4)$ & & 0.993 & $x$ \\
\hline Patients with T2D & $12(23.1)$ & & $18(12.1)$ & & 0.055 & $x$ \\
\hline Chronic kidney failure & $20(38.5)$ & & $61(40.9)$ & & 0.754 & $x$ \\
\hline Smokers & $8(15.4)$ & & $53(36.1)$ & & 0.005 & $x$ \\
\hline Patients treated with diuretics & $17(41.5)$ & & $24(27.3)$ & & 0.107 & $x$ \\
\hline
\end{tabular}

$\mathrm{t}$ - t-test; U - Mann-Whitney test; $\mathrm{X}$ - Chi-square test

Table 2. Serum levels of uric acid, use of diuretics and the distribution of conventional cardiovascular risk factors in patients with T2D and non-diabetic patients are displayed

\begin{tabular}{|c|c|c|c|c|}
\hline \multirow{2}{*}{ Index } & \multicolumn{2}{|c|}{$\begin{array}{l}\text { Patients with asymptomatic hyperuricemia } \\
\qquad(\mathrm{n}=52)\end{array}$} & \multicolumn{2}{|c|}{ Patients with gout $(n=149)$} \\
\hline & Patients with T2D & Non-diabetics & Patients with T2D & Non-diabetics \\
\hline $\begin{array}{l}\text { Serum level of uric acid }(\mu \mathrm{mol} / \mathrm{l}) \\
\text { Mean (SD) }\end{array}$ & $470.1(63.7)$ & $458.1(63.1)$ & $538(97.9)$ & $482.6(114.4)$ \\
\hline Use of diuretics n (\%) & $4(44.4)$ & $13(40.6)$ & $5(50.0)$ & $19(24.4)$ \\
\hline Dyslipidemia n (\%) & $9(90.0)$ & $29(85.3)$ & $11(78.6)^{*}$ & $100(97.1)$ \\
\hline Obesity n (\%) & $8(66.7)$ & $14(35.0)$ & $11(61.1)$ & $56(42.7)$ \\
\hline Arterial hypertension n (\%) & $11(91.7)^{*}$ & $24(60.0)$ & $17(94.4)$ & $102(78.5)$ \\
\hline Cardiovascular event n (\%) & $5(41.7)^{*}$ & $3(7.5)$ & $9(50.0)^{*}$ & $14(10.7)$ \\
\hline eGFR (< 90 ml/min) n (\%) & $5(41.7)$ & $15(37.5)$ & $5(27.8)$ & $56(42.7)$ \\
\hline Smokers n (\%) & $1(8.3)$ & $7(17.5)$ & $5(27.8)$ & $48(37.2)$ \\
\hline
\end{tabular}

${ }^{*} p<0.05$ 

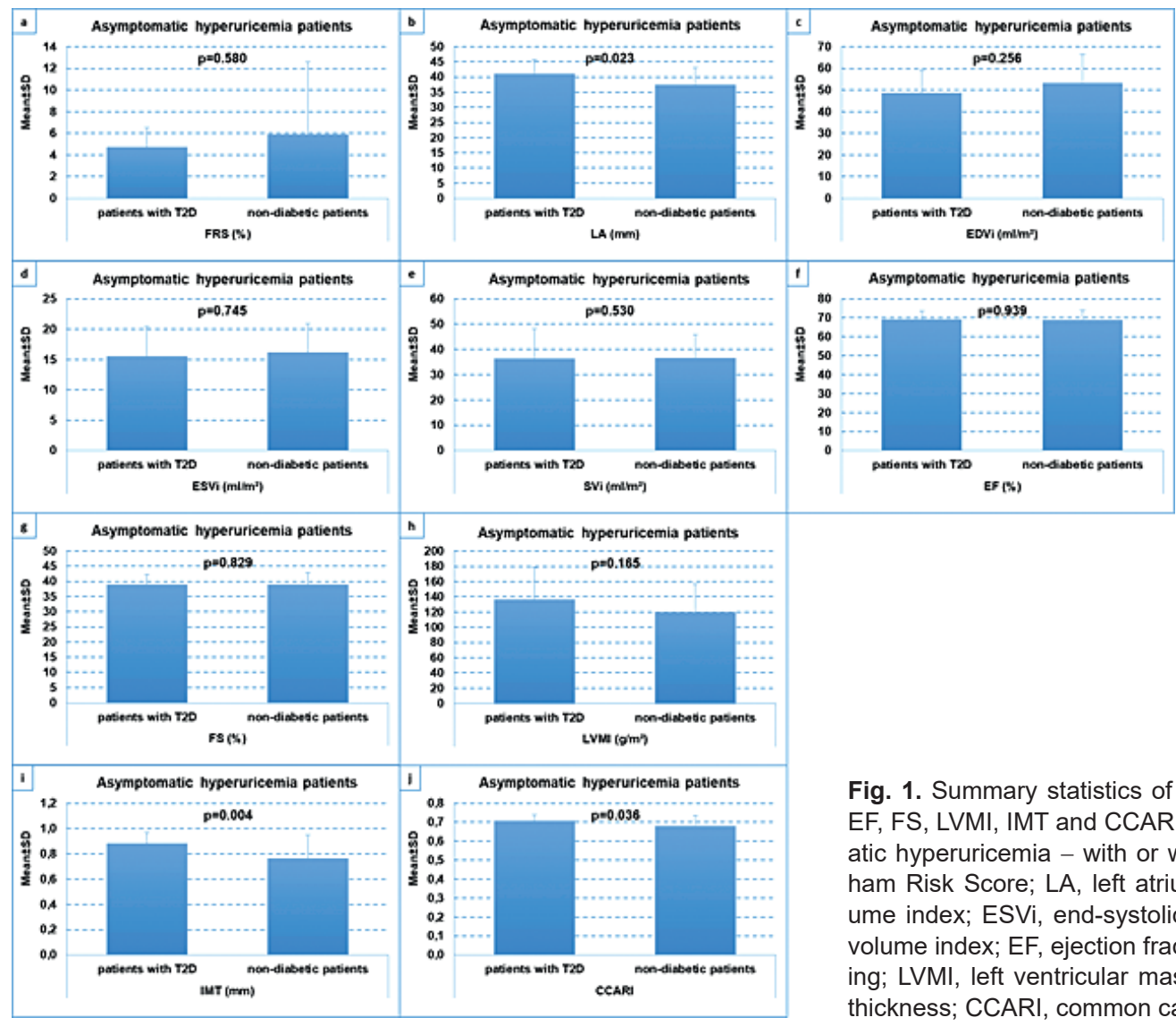

Fig. 1. Summary statistics of FRS, LA, EDVi, ESVi, SVi, $E F, F S, L V M I, I M T$ and CCARI in patients with asymptomatic hyperuricemia - with or without T2D. FRS, Framingham Risk Score; LA, left atrium; EDVi, end-diastolic volume index; ESVi, end-systolic volume index; SVi, stroke volume index; EF, ejection fraction; FS, fractional shortening; LVMI, left ventricular mass index; IMT, intima-media thickness; CCARI, common carotid artery resistive index
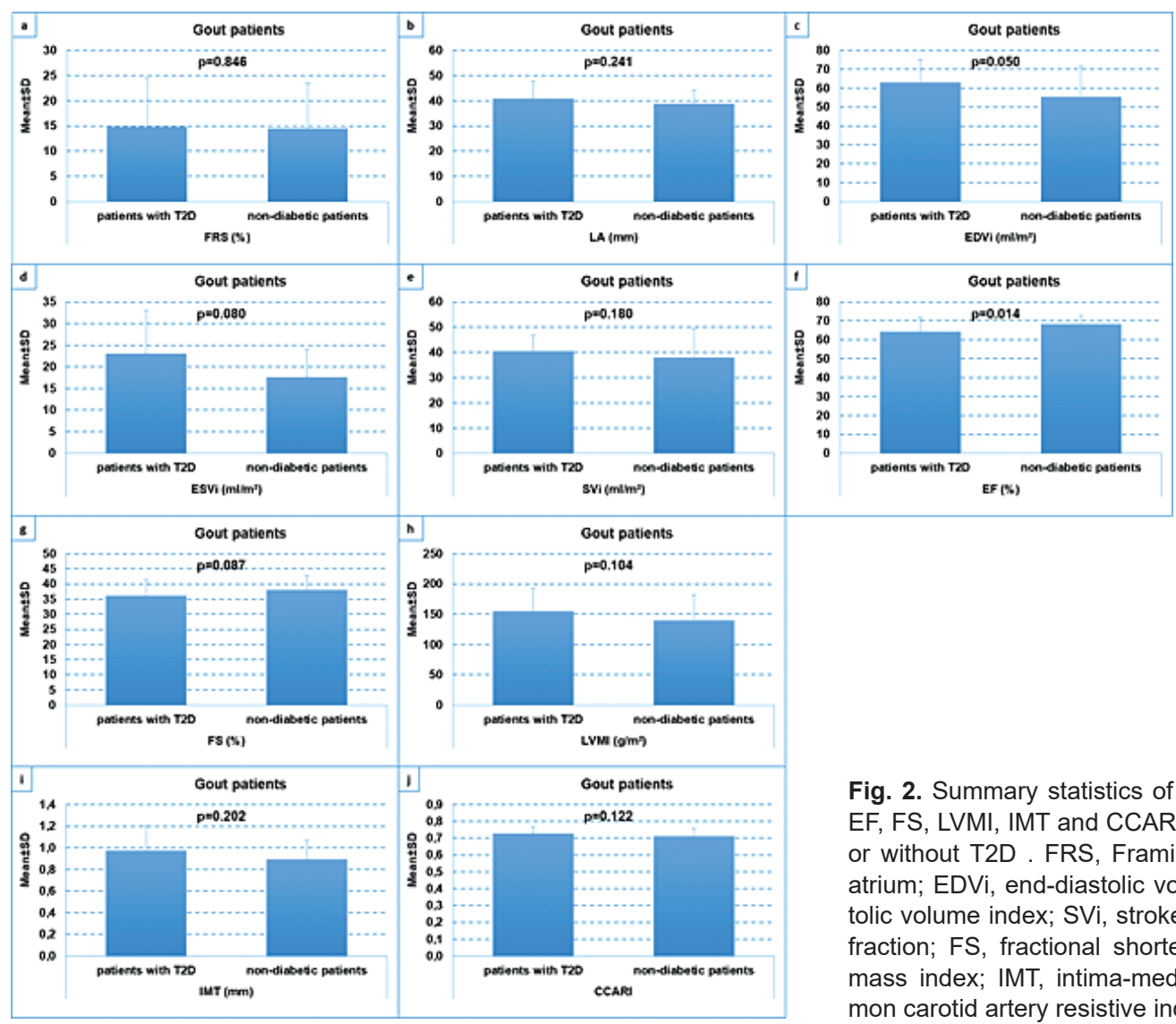

Fig. 2. Summary statistics of FRS, LA, EDVi, ESVi, SVi, $E F, F S, L V M I$, IMT and CCARI in patients with gout - with or without T2D . FRS, Framingham Risk Score; LA, left atrium; EDVi, end-diastolic volume index; ESVi, end-systolic volume index; $\mathrm{SVi}$, stroke volume index; $\mathrm{EF}$, ejection fraction; FS, fractional shortening; LVMI, left ventricular mass index; IMT, intima-media thickness; CCARI, common carotid artery resistive index 


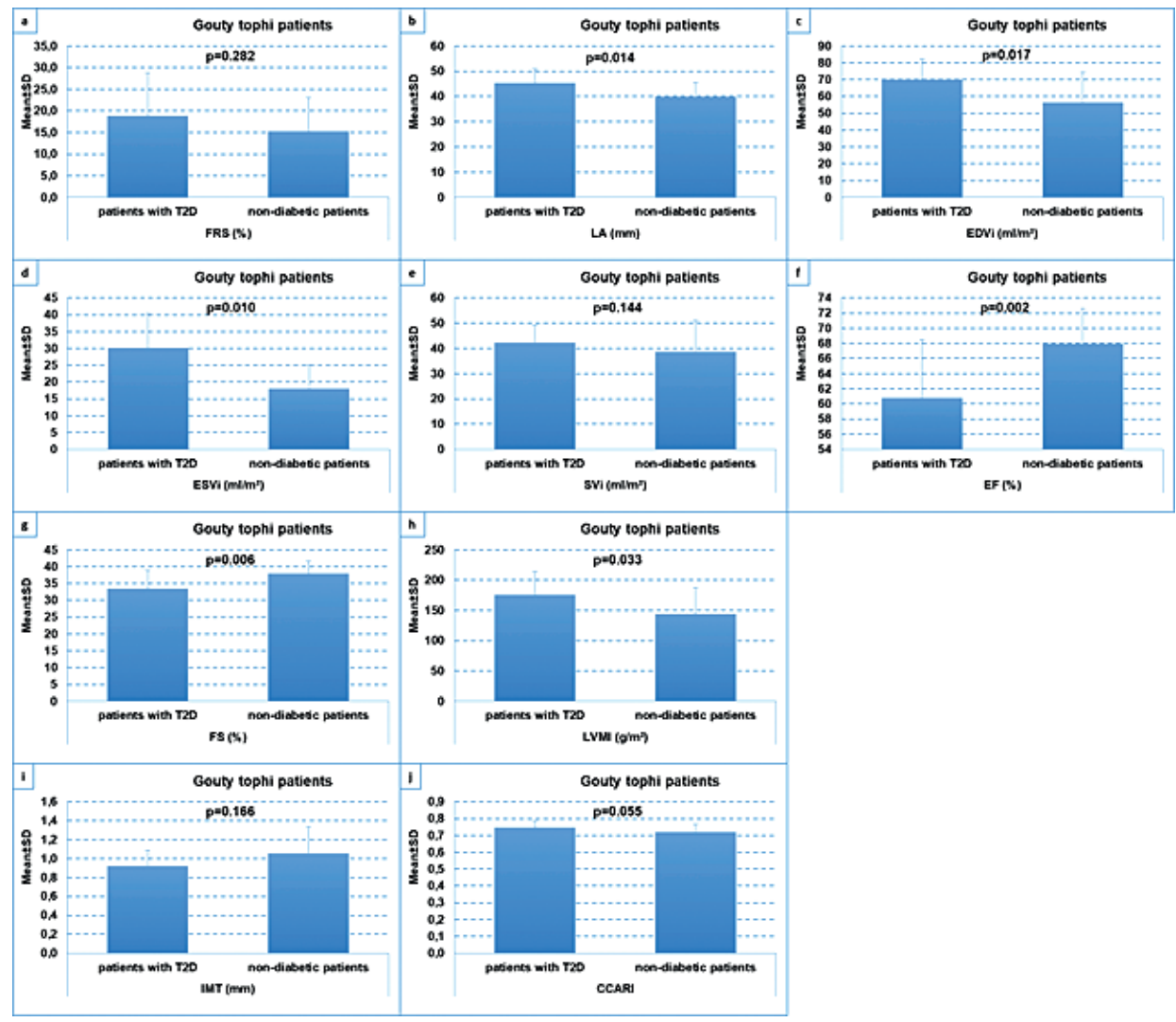

Fig. 3. Summary statistics of FRS, LA, EDVi, ESVi, SVi, EF, FS, LVMI, IMT and CCARI in patients with gouty tophi - with or without T2D. FRS, Framingham Risk Score; LA, left atrium; EDVi, end-diastolic volume index; ESVi, end-systolic volume index; SVi, stroke volume index; EF, ejection fraction; FS, fractional shortening; LVMI, left ventricular mass index; IMT, intima-media thickness; CCARI, common carotid artery resistive index

\section{DISCUSSION}

In the present study, we found that the mean values of the FRS among patients with and without T2D in the groups with asymptomatic hyperuricemia, gout and gouty tophi were not different. Involvement of the heart and common carotid arteries was more pronounced in individuals with T2D. In the group with asymptomatic hyperuricemia, the diabetic patients had larger LA and more advanced atherosclerotic and arteriosclerotic common carotid arteries changes. Subjects with gout and diabetes had a significantly lower systolic function in comparison to gout subjects without diabetes. These results led us to investigate the alterations in individuals with gouty tophi, as tophi maintain a chronic inflammatory process even during the intercritical phase [21]. We found that in individuals with gouty tophi and diabetes the morphological and functional heart parameters were more severely affected than in those without diabetes.

Previous echocardiography studies have established that a large proportion of individuals with T2D have left ventricular hypertrophy, diastolic dysfunction, ab- normally large left atriums and low EF [22]. Data from the literature also show that T2D and even the prediabetic state are independently associated with the presence of left ventricular hypertrophy [23]. On the other hand, evidence exists that gout and especially gouty tophi are linked to left ventricular diastolic dysfunction and left atrium volume enlargement [24]. As far as the carotid arteries are concerned, T2D leads to thicker intima-media, higher stiffness of the arterial wall and development of atherosclerotic plaques [25, 26]. Similarly, hyperuricemia and gout are associated with greater thickness and stiffness of the carotid arteries, as well as with a higher frequency of atherosclerotic plaques [27-29].

Uric acid induces mitochondrial oxidative stress in the hepatocytes and may lead to development of fatty liver. All this may worsen the insulin resistance by reducing the bioavailability of nitric oxide (NO) [11]. There is evidence that maintaining low-grade inflammation in gout patients unlocks the development of diabetes, with C-reactive protein (CRP), IL-6 and soluble adhesion molecules playing a major role. At the renal level, insulin increases the reabsorption of 
urate by activating URAT- 1 and reducing its excretion [31]. Based on our findings, we conclude that type 2 diabetes in combination with asymptomatic hyperuricemia, and especially with gouty tophi, creates a complex proatherogenic milieu with interlacing and mutually potentiating mechanisms. Further research is needed to elucidate these complex proatherogenic mechanisms.

In conclusion, we found no difference in the FRS between diabetics and non-diabetics in the examined groups, although by complex multimodal ultrasonography we could identify differences in the individual parameters of the heart and common carotid arteries. These results suggest that a more comprehensive approach is needed in the assessment of cardiovascular risk in type 2 diabetics with asymptomatic hyperuricemia and gouty tophi. Several limitations of our study deserve to be mentioned. This was a single centre cross-sectional study, laboratory parameters were measured at a fixed point of time, and in asymptomatic hyperuricemia no additional imaging methods for detection of silent monosodium urate crystals were applied. Future studies should be able to surpass these limitations and give a more comprehensive picture of the cardiovascular risk profile in type 2 diabetic patients with asymptomatic hyperuricemia and gout.

\section{Conflicts of interest}

The authors declare no conflicts of interest.

\section{REFERENCES}

1. Kim SY, Guevara JP, Kim KM, et al. Hyperuricemia and risk of stroke: a systematic review and meta-analysis. Arthritis Rheum. 2009; 61:885-892.

2. Zhu Y, Pandya BJ, Choi HK. Comorbidities of gout and hyperuricemia in the US general population: NHANES 2007-2008. Am J Med. 2012; 125(7):679-687.

3. Lai HM, Chen CJ, Su BY, et al. Gout and type 2 diabetes have a mutual inter-dependent effect on genetic risk factors and higher incidences. Rheumatology 2012; 51(4):715-20.

4. Carnethon MR, Fortmann SP, Palaniappan L, et al. Risk factors for progression to incident hyperinsulinemia: the Atherosclerosis Risk in Communities Study, 1987-1998. Am J Epidemiol 2003; 158(11):1058-67.

5. Boyle JA, McKiddie M, Buchanan KD, et al. Diabetes mellitus and gout. Blood sugar and plasma insulin responses to oral glucose in normal weight, overweight, and gouty patients. 1969; Annals of the Rheumatic Diseases; 28(4):374-8.

6. Niskanen L, Laaksonen DE, Lindstrom J, et al. Serum uric acid as a harbinger of metabolic outcome in subjects with impaired glucose tolerance: the Finnish Diabetes Prevention Study. Diabetes Care; 29(3):709-11.

7. Choi HK, De Vera MA, Krishnan E. Gout and the risk of type 2 diabetes among men with a high cardiovascular risk profile. Rheumatology 2008; 47(10):1567-10.
8. Kim SC, Solomon DH. Risk of incident atrial fibrillation in gout: a cohort study. Ann Rheum Dis. 2016; 75(8):1473-8.

9. Rho YH, Lu N, Peloquin CE et al. Independent impact of gout on the risk of diabetes mellitus among women and men: a population-based, BMI-matched cohort study. Ann Rheum Dis. 2016; 75(1):91-5.

10. Pan A, Teng GG, Yuan JM, Koh WP. Bidirectional Association between Diabetes and Gout: the Singapore Chinese Health Study. Scientific reports. 2016 May 10;6:25766. PubMed PMID: 27161168. Pubmed Central PMCID: 4861921.

11. Lanaspa MA, Sanchez-Lozada LG, Choi YJ et al. Uric acid induces hepatic steatosis by generation of mitochondrial oxidative stress: potential role in fructose-dependent and -independent fatty liver. J Biol Chem. 2012;287(48):40732-44.

12. Pontremoli $R$ The role of urate-lowering treatment on cardiovascular and renal disease: evidence from CARES, FAST, ALL-HEART, and FEATHER studies, Current Medical Research and Opinion, 2017,33:sup3, 27-32.

13. Kizer JR, Bella JN, Palmieri V et al. Left atrial diameter as an independent predictor of first clinical cardiovascular events in middle-aged and elderly adults: The Strong Heart Study (SHS). Am Heart J. 2006; 151(2):412-418.

14. Staub D, Meyerhans A, Bundi B, et al. Prediction of cardiovascular morbidity and mortality: comparison of the internal carotid artery resistive index with the common carotid artery intima-media thickness. Stroke 2006; 37:800-805.

15. Wallace SL, Robinson H, Masi AT, et al. Preliminary criteria for the classification of the acute arthritis of primary gout. Arthritis Rheum 1977, 20(3):895-900.

16. Expert Panel on Detection, Evaluation, and Treatment of High Blood Cholesterol in Adults. Executive Summary of the Third Report of the National Cholesterol Education Program (NCEP) Expert Panel on Detection, Evaluation, and Treatment of High Blood Cholesterol in Adults (Adult Treatment Panel III). JAMA 2001; 285:2486-97.

17. Grundy SM, Cleeman JI, Bairey Merz CN, et al. Implications of recent clinical trials for the National Cholesterol Education Program Adult Treatment Panel III guidelines. Circulation. 2004 Jul 13;110(2):227-39.

18. National Kidney Foundation K/DOQI clinical practice guidelines for chronic kidney disease: evaluation, classification, and stratification. Am J Kidney Dis 2002; 39:S1-S266.

19. Heine GH, Gerhart MK, Ulrich C, et al. Renal Doppler resistance indices are associated with systemic atherosclerosis in kidney transplant recipients. Kidney Int 2005; 68:878-885.

20. Lang RM, Beirig M, Devereux RB, et al. Recommendations for chamber quantification: a report from the American Society of Echocardiography's Guidelines and Standards Committee and the Chamber Quantification Writing Group, developed in conjunction with the European Association of Echocardiography, a branch of the European Society of Cardiology. J Am Soc Echocardiogr 2005; 18:1440-1463.

21. Dalbeth N, Pool B, Gamble GD, et al. Cellular characterization of the gouty tophus: a quantitative analysis. Arthritis Rheum 2010, 62(5):1549-1556.

22. Jørgensen PT, Jensen MT, Mogelvang R, et al. Abnormal echocardiography in patients with type 2 diabetes and relation to symptoms and clinical characteristics. Diabetes \& Vascular Disease Research 2016, 13(5) 321-330.

23. Negishi K. Echocardiographic feature of diabetic cardiomyopathy: where are we now? Cardiovasc Diagn Ther 2018;8(1):47-56. 
24. Lin JC, Lin CL, Chen MC, et al. Gout, not hyperuricemia alone, impairs left ventricular diastolic function. Arthritis Res Ther. 2015; 17: 323.

25. Kiechl S, Willeit J. The natural course of atherosclerosis. Part 1: incidence and progression. Arterioscler Thromb Vasc Biol. 1999;19(6):1484-1490.

26. Zhang L, Yin JK, Duan YY, et al. Evaluation of carotid artery elasticity changes in patients with type 2 diabetes. Cardiovasc Diabetol. 2014; 13: 39.

27. Cippoli JA, Ferreira-Sae MC, Martins RP et al. Relationship between serum uric acid and internal carotid resistive index in hypertensive women: a cross-sectional study. BMC Cardiovascular Disorders. 2012;12:52.
28. Çukurova S, Pamuk ÖN, Ünlü E, et al. Subclinical atherosclerosis in gouty arthritis patients: a comparative study. Rheumatol Int. 2012; 32(6):1769-73.

29. Montalcini T, Gorgone G, Gazzaruso C, et al. Relation between serum uric acid and carotid intima-meida thickness in healthy postmenopausal women. Intern Emerg Med. 2007; 2(1):19-23.

30. Tavil Y, Kaya MG, Oktar SO, et al. Uric acid level and its association with intima-media thickness in patients with hypertension. Atherosclerosis. 2008; 197(1):159-163.

31. Miao Z, Yan S, Wang J, et al. Insulin resistance acts as an independent risk factor exacerbating high-purine diet induced renal injury and knee joint gouty lesions. Inflamm Res. 2009; 58(10):659-68. 